\title{
An Improved Hybrid Energy Efficient Clustering Technique to Enhance the Lifespan of Wireless Sensor Networks
}

\author{
Deepa V. Jose \\ Christ University \\ Hosur RoadBangalore
}

\author{
G. Sadashivappa \\ $R \mathrm{~V}$ College of Engineering \\ Mysore Road \\ Bangalore
}

\begin{abstract}
Clustering is a significant mechanism used in Wireless Sensor Networks in order to have an efficient energy balance which is inevitable to prolong the lifetime. The concept of unequal clustering has proved to be an effective method for load balancing and thereby reducing hotspot issues in the energy constrained wireless sensor networks. This paper proposes an energy efficient clustering mechanism which can enhance the lifetime of Wireless Sensor Networks. The proposed protocol is an enhancement of the Hybrid Energy-Efficient Distributed clustering protocol where the clustering is performed using energy efficient varying sized clustering algorithm. An overview of the Hybrid Energy-Efficient Distributed clustering protocol, highlighting its advantages and drawbacks is also given in this paper. The performance of the proposed algorithm is evaluated through simulation using MATLAB based on the parameters delay in packet delivery, residual energy of the network and the number of live nodes during precise time periods. Extensive simulation results show that the proposed enhanced Hybrid Energy-Efficient Distributed clustering algorithm gives better performance compared to the energy efficient clustering protocols like Hybrid EnergyEfficient Distributed clustering protocol and Unequal Hybrid Energy-Efficient Distributed clustering protocol based on the above mentioned parameters.
\end{abstract}

\section{General Terms}

Energy Efficiency, Lifetime Enhancement, Wireless Sensor Networks

\section{Keywords}

Energy Efficiency, HEED, Unequal Clustering

\section{INTRODUCTION}

There exists a number of tiny resource constrained wireless nodes in Wireless Sensor Networks(WSNs) that are deployed in an environment either arbitrarily or in a specific manner in order to monitor some physical phenomena like temperature, humidity and so on. Enormous applications pertaining to our daily lives is tailored by the use of WSNs especially in areas of agriculture, health and medical services, rescue and surveillance applications and so on. Extending the lifetime of these resource constrained networks is inevitable so as to exploit the benefits of this technology.

Most of the energy in WSNs is consumed for sensing, processing and communication wherein communication of the sensor nodes contributes to the major energy consumption. Selection of a routing strategy in WSNs is more challenging than other types of networks due to its specific characteristics and it should ensure the delivery of packets to the destination with the minimum utilization of the available resources so as to extend the network lifetime[1]. So devising an energy efficient routing strategy is necessary to prolong the network lifetime. Several policies are adopted for energy efficient route selection like call packing, load balancing, minimum hop routing, etc. Different types of routing protocols are designed based on the network structure. In this, the hierarchical routing protocols aims in the energy efficient routing which is a mandate to extend the network lifetime. These categories of protocols use the clustering concept. Clustering is the process of dividing the entire monitored area into different segments based on certain criteria. This avoids the need of all sensor nodes to transmit the sensed information directly to the base station. Instead a representative called cluster head from each cluster will aggregate the data from all sensor nodes in that specific cluster and route it to the base station. The clustering technique, thereby saves energy to a great extent [1-5]. The general scenario of clustering is depicted in Figure 1.

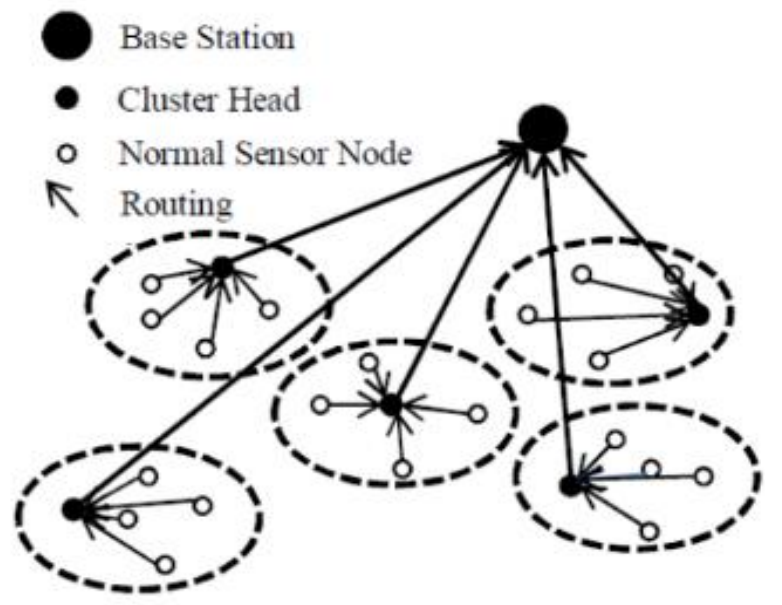

Fig 1:Clustering scenario in WSNs[2]

There are different types of clustering, based on the mechanism adopted for clustering as well as the selection of cluster heads. The main challenges in designing a clustering mechanism lies in finding the optimal cluster size and the number of cluster heads, election of cluster head and reelection, and the maintenance of the entire cluster[3]. The major classifications of clustering algorithms are probabilistic clustering algorithms and non-probabilistic clustering algorithms based on the approach of cluster head selection. The probabilistic clustering algorithms, where the cluster head selection is based on the probability concept, are known for their efficient network utilization and network management which embraces load balancing and network lifetime elongation. Examples of clustering algorithms under this category includes LEACH and Two Level Low Energy 
Adaptive Clustering Hierarchy ( $\mathrm{T}$ - LEACH). In nonprobabilistic method the cluster head selection is based on other parameters like cluster heads distance from the base station, connectivity etc. The various weight-based clustering algorithms and biologically inspired clustering algorithms belongs to this category [2-3]. Data gathering and aggregation in a hierarchical manner is a common energy saving mechanism adopted in WSN applications.

In clustered Wireless Sensor Networks, the cluster heads will have to spend more energy because of intra cluster and inter cluster routing and gets depleted at the earliest. Similarly, the nodes near the sink also faces the same problem because of their frequent participation in data transmission. In clustering, clusters formed will be usually of uniform sizes because of the radio range. In all such cases the problems of load imbalance and hotspot issues arises which is a threat for the lifetime of the network. Majority of the clustering techniques adopts clusters of equal size which exacerbates the hot spot issue. Unequal clustering assures the avoidance of the same to a better outspread[4][5].

The main contribution of this paper is the use of an unequal clustering strategy in the Hybrid Energy-Efficient Distributed clustering. The proposed approach proves to be better in enhancing the lifetime of the network. The issue of overburdening the cluster heads near the base station is avoided by varying the cluster size. This eliminates the early energy depletion of the nodes near the base station there by prolonging the lifetime of the network.

The organization of the paper is as follows; Section 2 gives an overview of various unequal clustering techniques. Section 3 gives a detailed review of HEED, highlighting the drawbacks. Section 4 describes the proposed enhanced HEED followed by a performance evaluation in Section 5. Section 6 mentions the advantages of the proposed approach and an insight towards the future work.

\section{RELATED WORK}

Hybrid Energy-Efficient Distributed clustering (HEED) proposed by Younis and Fahmy [6] is an efficient clustering algorithm which enhances the lifetime as well the scalability of the network by a different approach for cluster head selection. It uses the parameters residual energy as well as the node degree for this process and attains a uniform cluster head distribution throughout the network resulting in a better load balance.

A vital concern in WSNs is efficient use of energy to maximize the network lifetime. To accomplish this motive balanced energy consumption among the nodes is inevitable which can be achieved through unequal clustering[4]. Selvi and Manoharan in [4] have done a detailed survey of the different unequal clustering algorithms like Energy Efficient Unequal Clustering, Multihop Routing Protocol with Unequal Clustering, Unequal Hierarchical Energy Efficient Distributed Clustering, Energy Efficient Distributed Unequal Clustering, Energy Driven Unequal Clustering, Unequal Cluster Based Routing and Unequal Clustering Size, mentioning their advantages and drawbacks .

A similar study is done in [5] by Wankhade and Choudhari along with a proposal for an innovative unequal clustering method. According to the authors energy preservation can be achieved by making clusters near base station with smaller size, compared to that of farther ones. They also experimented with unequal inter clustering which gave better results than equal sized clusters in terms of energy consumption.
Lee et.al in [7] proposes a novel distributed unequal clustering algorithm which introduces a new waiting time for reclustering. A combinational function of number of neighbouring nodes and residual energy is used for formulating waiting time. The author's claims through simulation that the proposed approach works better in cluster head distribution and balancing of energy consumption.

Dhanpal, Joseph, and Panicker in [8] propose an algorithm for unequal clustering and a routing protocol for wireless sensor networks with non-uniform node distribution. They consider the cases of homogeneous and heterogeneous node distribution. According to them the simulation results proves to solve the hot spot issues and balances the cluster energy consumption leading to better life time. They have compared their proposed algorithm with another unequal clustering algorithm called EADC. The authors propose a new design of cluster radius resulting in an improved clustering method where the clustering is done in an unequal manner. The simulation results show that this approach could balance the load among the clusters. They also construct a routing tree for inter-cluster communication.

The topology of the WSN as well the node distribution will be always varying. To support the uneven node distribution and different scenario of network topology Chen et.al in [9] propose an improved distributed unequal clustering protocol. A new competition radius for clusters is experimented in this for unequal clustering. Through simulation and theoretical analysis, the authors claim that the proposed protocol is able to balance the energy and extend the lifetime of the network.

Another unequal clustering mechanism is given in [10] by Hassan and Hamed where the optimal number of nodes in a cluster is calculated. The authors also suggest that the clusters near the base station should not be overburdened with larger number of nodes so that they can save energy for inter cluster communication. Simulation results shows that the proposed algorithm improves the nodes lifetime.

A proposal for enhancing energy efficiency which is location based is mentioned in [11] by Kumar and Kumar. The authors use the rotation of the cluster heads to balance the energy consumption. This work treats clusters as virtual concentric circles. For the initial round the cluster head is the chosen as the one which lies in the mid-way of the any two concentric circles. Periodic rotation of the cluster head is also performed so as to balance the energy among the nodes.

An unequal clustering algorithm is given by Aierken et.al in [12] which also uses the concept of rotation of the cluster head. The authors in this article propose a novel strategy of rotating the cluster head in a cluster using unequal clustering protocol, RUHEED, which solves the hot spot issues. The experiment results show that RUHEED gives better network life span compared to other clustering algorithms like HEED.

Chen and Wang gives a different approach for selecting the coordinator node within a cluster. Two approaches are mentioned where the coordinator node can be selected randomly or based on energy level. The method of routing carried out is also aggregation based and non-aggregation based. This study gives a comparison of these different strategies though numerical methods and simulation[13] .

A proposal for weight based cluster head selection is given in [14] by Mahajan,Malhotra and Sharma. The clustering mechanisms aims at energy saving as well as load balancing. The proposed method is compared with LEACH, WCA and IWCA based on consumption of energy and lifetime. Here the 
cluster head selection is based on minimum path loss factor, average energy and the node degree. In this research work, a study on the impact of local clustering and base station location is also carried out.

A cluster head selection based on weighted probability is carried out in [15] by Kumar,Aseri, and Patel. Here heterogeneous sensor nodes are deployed in a random manner. The optimal cluster head selection is carried out in an analytical manner. Each node can elect itself as cluster head based on the energy level and the weighted probability. The non-cluster members send the sensed data to the cluster head and cluster head communicates it to the base station through single hops which reduces the energy usage. The proposed approach is compared with LEACH, DEEC and DT and proves to be more stable and energy efficient.

Wang, $\mathrm{Li}$ and Zhou in [16] propose a dynamic clustering method where the cluster head selection is based on joint weight model. According to the authors, the simulation results shows that the proficiency of the proposed approach based on data traffic and lifetime.

Alabass and Elleithy in [17] propose a mathematical model that helps to evaluate the residual energy level of each node in a cluster to elect the cluster head. The residual energy is calculated taken in to consideration, the energy spent for data transmission from cluster nodes to the cluster heads, from the cluster head to the base station and for the scheduling purpose.

\section{HEED: A HYBRID ENERGY EFFICIENT DISTRIBUTED CLUSTERING ARCHITECTURE}

HEED uses a distributed clustering architecture where the clustering is done in a specific number of iterations. The clustering problem defined by HEED is to identify a set of cluster heads which can cover the entire field, which is deployed with $n$ sensor nodes, and each node must belong to exactly one and only one cluster. Each node should be able to communicate with the cluster head in single hop. Wherein the clustering is performed in iterations of fixed numbers, the nodes will be assigned the role of either cluster head (considering nodes with high residual energy and high proximity to the base station) or a regular node. It uses a probabilistic approach for cluster head selection. The main feature of HEED is that it feats the convenience of multiple transmission power levels at sensor nodes. The clusters are formed so as to have maximum load balance and increased life time. This makes HEED flexible to apply for many applications especially those which require scalability, fault tolerance and extended life time[6].

\subsection{Network Model}

According to Hybrid Energy-Efficient Distributed clustering protocol, the following assumptions are made for the sensor network.

- All the sensor nodes are quasi-stationary.

- Symmetric links are used for communication.

- All nodes are unaware of their location.

- The significance and capabilities of all the nodes are similar.

- The batteries cannot be re-charged as they are left unattended after deployment.
- The number of transmission power levels are fixed for each node.

\subsection{HEED Protocol}

The HEED protocol works in three main phases;

- Initialization

- Main processing

- Finalization

In the initialization phase, the sensors put their probabilities to become $\mathrm{CHs}$, and in the main processing phase the sensors go through various procedures to elect the $\mathrm{CHs}$. It is in the finalization phase each sensor join the least communicationcost $\mathrm{CH}$ or announce itself as a $\mathrm{CH}$. The re-clustering in HEED is dynamically occasioned at the beginning of each round which is a pre- defined period of time which is application dependent.

Suppose $T_{C P}$ is the duration for clustering and $T_{N O}$ is time interval for network operation. New clustering and cluster head selection is elicited every $T_{C P}+T_{N O}$ seconds. The clustering process requires a number of iterations $N_{i t e r}$, at each node. For each step a time $t_{c}$, should be given which is sufficient to receive messages from any neighbor within the range of the cluster. In the initial stage the probability of cluster heads $C_{\text {prob }}$ is set to be $5 \%$ of the total nodes and in later stages the probability of becoming $\mathrm{CH}_{\text {prob }}$, is calculated as follows:

$$
C H_{\text {prob }}=C H_{\text {prob }} \times \frac{E_{\text {resi }}}{E_{m}}
$$

A predefined value $C_{p r o b}$ is the initial probability, $E_{\text {resi }}$ is the residual energy and $E_{m}$ is the maximum energy of the sensor nodes. Later comes the iterative phase or main processing phase where the competition among tentative cluster heads to become the next new cluster heads happens. The one with the most residual energy and in case of any ties, the one with the most node degree or node proximity to the base station will be selected. In the final phase, nodes which are not cluster heads joins the $\mathrm{CH}$ which is nearest to them or declares itself as $\mathrm{CH}$ in case they could not find any $\mathrm{CH}$ to join.

The main advantage of HEED is that it does not allow any node to be in more than one cluster. It could achieve a uniform distribution of cluster heads throughout the network; but terminates after a fixed number of iterations and reclustering has to be initiated again. HEED also doesn't look into the hotspot problem which is a major threat for the networks life time.

\section{PROPOSED ENHANCED HEED ALGORITHM}

\subsection{Network Model}

A set of $\mathrm{n}$ sensor nodes are deployed in a square region of $\mathrm{A} \times$ $\mathrm{A} \mathrm{m}{ }^{2}$ randomly. The base station is located at the left top side of the deployed area. Once the deployment of the sensor nodes is over, they are assumed to be stationary. Based on the received signal strength, the approximate distance between the nodes can be calculated. The radio energy dissipation model assumes error free communication links and uses both free space and multipath channel model mentioned by Younis and Fahmy in [6]. In order to run the transmitter and the receiver circuitry, the electronic energy spent by the sensor node $E_{\text {elec }}=50 \mathrm{~nJ} / \mathrm{bit}$. $\mathrm{E}_{\mathrm{a}}$, the energy spent by the transmitter amplifier is dependent on the distance ' $\mathrm{d}$ ' between the sender and the receiver. i.e. $\mathrm{E}_{\mathrm{a}}=\mathrm{E}_{\mathrm{f} \mathrm{s}}$ for a free space model when 
$\mathrm{d}<\mathrm{d}_{0}$ and $\mathrm{E}_{\mathrm{a}}=\mathrm{E}_{\mathrm{mf}}$ for a multipath model when $\mathrm{d} \geq \mathrm{d}_{0}$, where $\mathrm{d}_{0}=75 \mathrm{~m}$ is a constant distance. $\mathrm{E}_{\mathrm{f} \mathrm{s}}=10 \mathrm{pJ} / \mathrm{bit} / \mathrm{m}^{2}$ and $\mathrm{E}_{\mathrm{mf}}=0.0013 \mathrm{pJ} / \mathrm{bit} / \mathrm{m}^{4}$. Based on the above assumptions for the radio model, for transmitting a k-size packet over a distance $\mathrm{d}$, the amount of energy consumed for transmission $\mathrm{E}_{\mathrm{Tx}}$, can be calculated as:

$E_{T X}=\left(E_{\text {elec }} \times k\right)+\left(E_{a} \times k \times d^{n}\right)$

where, $\mathrm{n}$ is assigned 2 for the free space model and 4 for the multipath model. The energy spent to receive a k-bit size message $\mathrm{E}_{\mathrm{Rx}}$ is calculated as:

$E_{R X}=E_{\text {elec }} \times k$

\subsection{Procedure}

The proposed enhanced HEED algorithm is focused on enhancing the network life time by lessening the hotspot problem by unequal clustering. So balancing of relay traffic and distributed clustering is the prime focus; considering the residual energy of node and the number of neighbor's node. It consists of three phases; the initiation, main processing and finalization phase.

- Initialization phase- initial network setup and clustering and specific waiting time for re-clustering

- Main processing phase- unequal clustering and $\mathrm{CH}$ rotation based on demand

- Finalization phase-clustering and data transmission

The initialization phase starts with the base station broadcasting a signal. All nodes calculate their distance to the base station based on the received signal strength. Within its communication range ' $r$ ', each node transmits a message consisting of its id and the residual energy. The average residual energy[9] of the neighbor nodes is calculated using the formula;

$E_{i a}=\frac{1}{n} \times \sum_{j=1}^{n} E_{j r}$

where $n$ denotes the total number of neighbor nodes and $E_{j r}$ is the residual energy of the neighbor nodes. A waiting time $t_{i}$ is also set for broadcasting the cluster election message in such a way that no two nodes will send this message at the same time. In order to have this a random value between $[0.9,1]$ is assigned to $\mathrm{V}_{\mathrm{r}}$. The waiting time[9] is calculated as;

$t_{i}= \begin{cases}\frac{E_{i a}}{E_{i r}} T_{2} V_{r}, & E_{i r} \geq E_{i a} \\ T_{2} V_{r}, & E_{i r}<E_{i a}\end{cases}$

Where $E_{i a}$ is the average residual energy, $E_{i r}$ is the residual energy of node $i, T_{2}$ is the time duration and random value between $[0.9,1]$.Initial steps of the proposed enhanced HEED is similar to HEED as in [6]. The entire operation happens in different rounds. Nodes will be aware of its residual energy, energy level of neighbor nodes and number of neighbor nodes. The average minimum rechargability power (AMRP) is calculated. Every round will be initiated with the clustering mechanism leading to clustering and later to data transmission through intra-cluster and inter-cluster mechanisms. After the initial round, a random period of waiting time is introduced as in (5) for initiating the unequal clustering. In HEED every $\mathrm{CH}$ uses the same competition radius irrespective of its distance from the base station which forms equal sized clusters which results in hotspots leading to energy depletion of $\mathrm{CHs}$ mainly near the base station. In order to avoid this issue, the proposed enhanced HEED uses an unequal clustering method defined in [9] for clustering where the cluster radius is calculated as;
$R_{c i}=\left[1-\propto\left(\frac{d_{m}-d_{i}}{d_{m a}-d_{m i}}\right)-\beta\left(\frac{E_{i}}{E_{m}}\right)\right] R_{m}$

where $d_{m a}$ and $d_{m i}$ are the maximum and minimum distance of the sensors from the base station, $d_{i}$ is the distance of the sensor nodes to the base station, $\alpha$ is a random value which belongs to $[0,1], R_{m}$ is the maximum value of available competition radius which is a pre-defined value, $\beta$ is a real random value in the interval $[0,1]$ and $E_{i}$ is the residual energy of the node $i$. The competition radius of a node is dependent on $E_{i}$ and $d_{i}$; the larger the values of $E_{i}$ and $d_{i,}$ the larger will be the competition radius. For the simulation $R_{m}$ is taken as the diagonal distance divided by ten.

Each node will have information about its energy level and the distance of it from the cluster head. Based on the message received from the cluster heads, distance will be calculated and the non-cluster head nodes will join the nearest cluster head. The different control message used the head_ message (selfid), join_message (selfid, headid), schedule_ message (order) and route message (selfid, selfenergy, disttoBS). For the simulation purpose, a fixed packet size of 256 bits is used. The common format of the packets used consists of a header field, payload field and a trailer, as shown Figure 2. Generally, the header field includes the current segment number, total number of segments in the higher layer packet, higher layer packet identifier and the source and destination identifiers. The payload contains information bits. The trailer contains the parity bits for error control[18].The packet length can be variable or fixed. It has an influence on the complexity of data transmission.

\begin{tabular}{|l|l|l|}
\hline Header & Payload & Trailer \\
\hline
\end{tabular}

\section{Fig 2: Format of link layer packet}

The packet reception rate and the transmission efficiency affects the good put and the energy efficiency. Finding the optimum packet therefore is very crucial in performance enhancement but cannot be generalized and is application dependent. The packet size is based on the application and the network used[18]. An example of the packet format used by the ZigBee network is represented in Figure 3 given below.

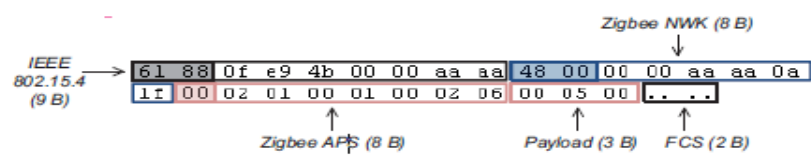

Fig 3: Format of ZigBee data packet

The aim of this research work is to avoid the premature death of the cluster heads near the base station by reducing the overload in them. Using the above mentioned cluster radius as in equation (6), the clusters near the base station will be small and obviously the cluster heads have to manage less number of nodes in the cluster saving the energy for forwarding the data. The flow chart mentioning the concept of the proposed enhanced HEED is given in Figure 4. 


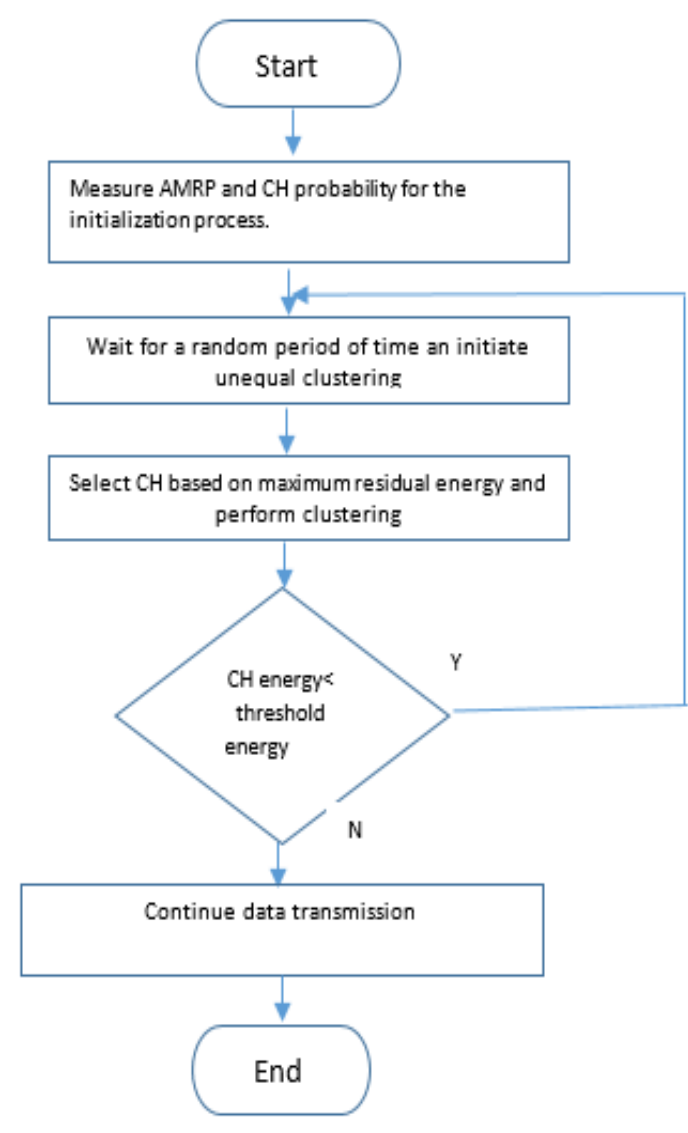

Fig 4: Flowchart of the proposed enhanced HEED algorithm

The steps in the proposed enhanced HEED can be summarized as follows.

Step1: Initial round cluster heads are constructed according to HEED algorithm.

Step 2: In the next round re-clustering is done based on the competition radius given in equation (6) based on the waiting time given in equation (5).

Step 3: Within a cluster the $\mathrm{CHs}$ will be rotated based on the residual energy set to a threshold value.

Step 4: If the CHs energy level goes beyond the threshold hold value, re-clustering is performed; go to Step 2.

Step 5: After $\mathrm{CH}$ selection and clustering is done, data is routed (intra-cluster and inter-cluster) to the base station.

\subsection{HEED Versus Proposed Enhanced HEED}

The main difference in HEED and the proposed enhanced HEED is in the method of clustering. While HEED creates clusters of equal size, the proposed enhanced HEED uses the unequal clustering technique, energy efficient varying size clustering algorithm. This approach eliminates the premature death of the nodes near the base station which will be continuously participating in the data transmission to the base station. Proposed enhanced HEED creates clusters of varied sizes and make sure that the clusters near the base station is small. As the cluster heads in smaller clusters require to coordinate lesser cluster members, the energy of the cluster heads can be preserved for forwarding the data. This in turn reduces the load on the cluster heads, avoids their energy depletion at a faster rate and thereby enhances the life time of the network.

\section{PERFORMANCE ANALYSIS}

For the comparison of the proposed enhanced HEED the parameters are set to be same as that in HEED[6]. The simulation happens in rounds where each round indicates a specific time duration (say 100s, 200s etc.). The area of simulation and the number of nodes deployed are also varied for performance analysis of the proposed approach. The simulation parameters for the case where the area of simulation is $100 \times 100$ meters and the number of nodes deployed are 50 is given in Table 2.

Table 2. Simulation parameters

\begin{tabular}{|l|l|}
\hline Parameters & Values \\
\hline Area of simulation & $100 \mathrm{~m} \times 100 \mathrm{~m}$ \\
\hline Base station location & $(20,80)$ \\
\hline Number of nodes & 50 \\
\hline $\mathbf{E}_{\text {elec }}$ & $50 \mathrm{~nJ} / \mathrm{bit}$ \\
\hline $\mathbf{E}_{\mathrm{fs}}$ & $10 \mathrm{pJ} / \mathrm{bit} / \mathrm{m}^{2}$ \\
\hline $\mathbf{E}_{\mathbf{m p}}$ & $0.0013 \mathrm{pJ} / \mathrm{bit}^{2} \mathrm{~m}^{4}$ \\
\hline Initial energy & $5 \mathrm{~J}$ \\
\hline Deployment model & Random \\
\hline
\end{tabular}

The different parameters used for performance evaluation of the proposed enhanced HEED are the lifetime of network, average residual energy of the network and average delay in packet delivery. The lifetime of the network can be calculated based on different parameters like the time when the first node dies or the time till the last node dies or when a certain percentage of the nodes get depleted. For the lifetime comparison here, the time till the entire nodes dies off is considered. Here the simulation of the data transmission and reception is done through energy consumption. Usually for the calculation of the delay for packet delivery, the sum of different delays like queuing delay, processing delay and transmission delay can be considered. Packet delay is the time since the packet is generated till it reaches the destination.

\subsection{Calculation of Average Residual \\ Energy, Delay and Number of Live \\ Nodes Based on the Simulation Where the Imagined Situation is Random Event Sensing}

Packets are generated randomly by every node. For calculating the average residual energy of the network for a particular round, a fixed amount of energy is reduced, for each packet generation, communication to the cluster head and cluster heads to the base station. When the specific round is over, the aggregate remaining energy of the nodes are calculated which is divided by the total number of nodes to get the average residual energy.

Each time a node randomly generates a packet, a counter is set to find the total number of packets generated for a particular round. The number of packets reaching the destination is also tracked. The average time taken by those successful packets to reach the destination is calculated which is the average delay in packet delivery. 
A threshold energy level is set for the nodes to check the status of the nodes. If the energy level of the node goes below the threshold level, that node is assumed to be dead. The count of the number of nodes who have energy above the threshold level gives the number of live nodes for that particular round.

The base station is fixed at the left side of the simulation area; in this case, specifically at $(20,80)$. The simulation is done for 500 rounds. The results represent an average of 10 rounds under the same simulation environment. The plain circles represent the normal nodes, the red color circle indicates the base station and the green colors indicate the cluster heads.

Scenario: Area of Simulation: $100 \mathrm{~m} \times 100 \mathrm{~m}$, Number of nodes deployed: 50

Figure 5 represents the random deployment of 50 sensor nodes over $100 \mathrm{~m} \times 100 \mathrm{~m}$ and Figure 6 gives cluster formation of proposed enhanced HEED.

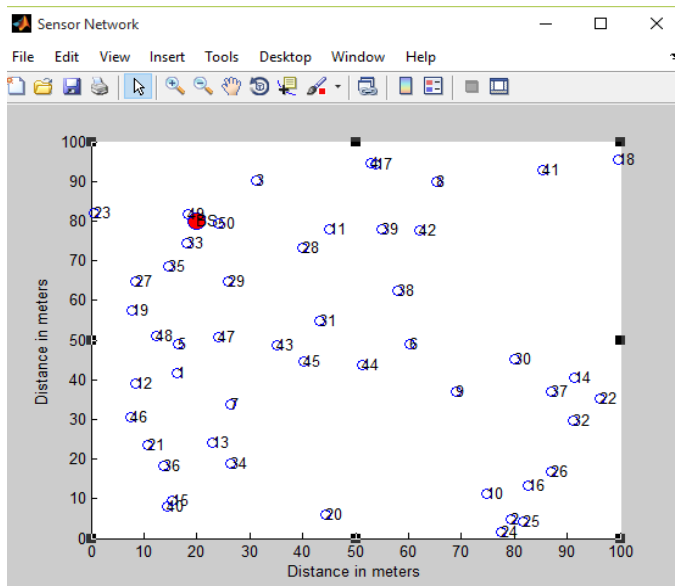

Fig 5: Random deployment of 50 sensor nodes in an area $100 \mathrm{~m} \times 100 \mathrm{~m}$

The number of live nodes at the end of the specific rounds is calculated. $\mathrm{N}$ represents the number of nodes deployed and $\mathrm{A}$ represents the area of deployment.

Figure 7 represents the comparison of the number of live nodes for the deployment scenario which is taken as 50 nodes deployed over $100 \mathrm{~m} \times 100 \mathrm{~m}$ area. Keeping the area of deployment constant different number of nodes are deployed in order to check the behavior of the sensor network.

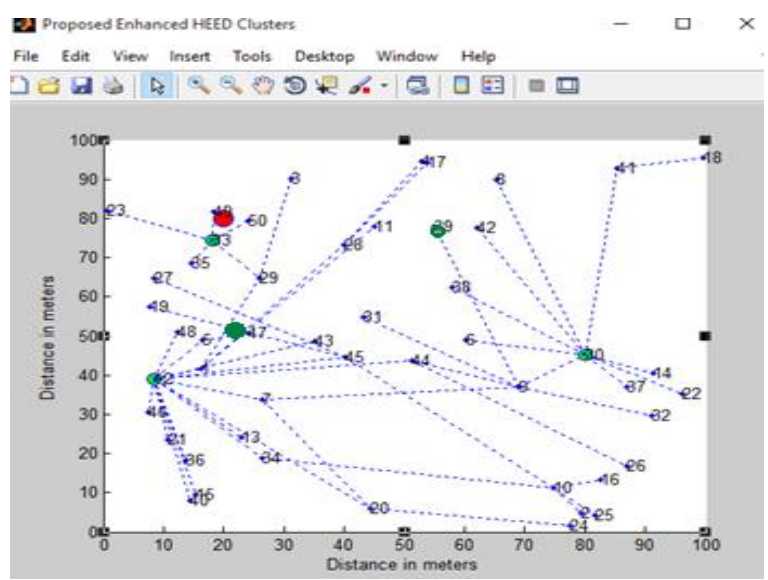

Fig 6: Cluster formation of Proposed enhanced HEED (Area of Simulation $=100 \mathrm{~m} \times 100 \mathrm{~m}$ and number of sensor nodes is 50)

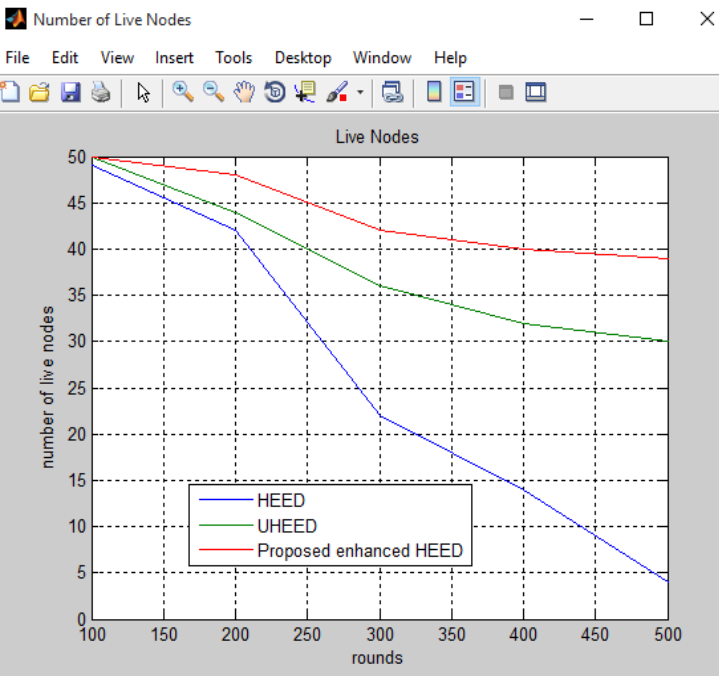

Fig 7: Number of alive nodes versus rounds $(\mathrm{N}=50$ and $A=100 \mathrm{~m} \times 100 \mathrm{~m}$ )

Based on Figure 7 it is evident that the number of live nodes in each round is more in the case of proposed enhanced Hybrid Energy-Efficient Distributed clustering protocol compared to Hybrid Energy-Efficient Distributed clustering protocol and Unequal Hybrid Energy-Efficient Distributed clustering protocol. In the case of HEED there is a drastic reduction in the number of live nodes as the rounds increases in all three scenarios. In UHEED there is a gradual depletion of nodes. Compared to these, the proposed enhanced algorithm retains the number of nodes alive for a longer time. In a similar scenario, the average delay in packet delivery is calculated. Figure 8 shows the simulation result for the case where 50 nodes are deployed in a $100 \mathrm{~m} \times 100 \mathrm{~m}$. Only the number of packets that successfully reach the destination is considered for calculating the delay. The time since their generation to the time it is received by the base station is calculated for each scenario. The proposed enhanced HEED shows less delay in packet delivery in all the cases. Simulation results for calculating the average residual energy of the nodes in the network is given below in Figure 9 where the residual energy of proposed enhanced HEED is higher compared to others.

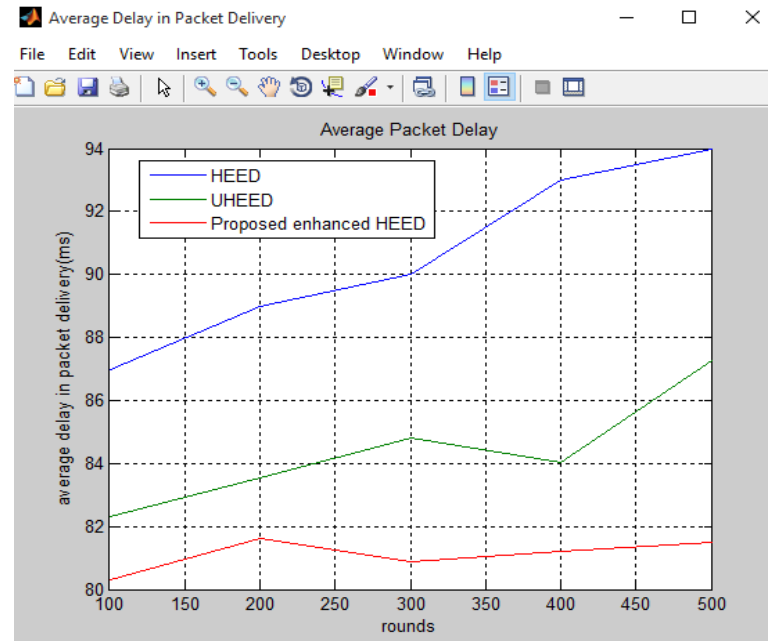

Fig 8: Average delay in packet delivery versus rounds

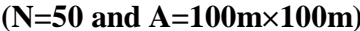




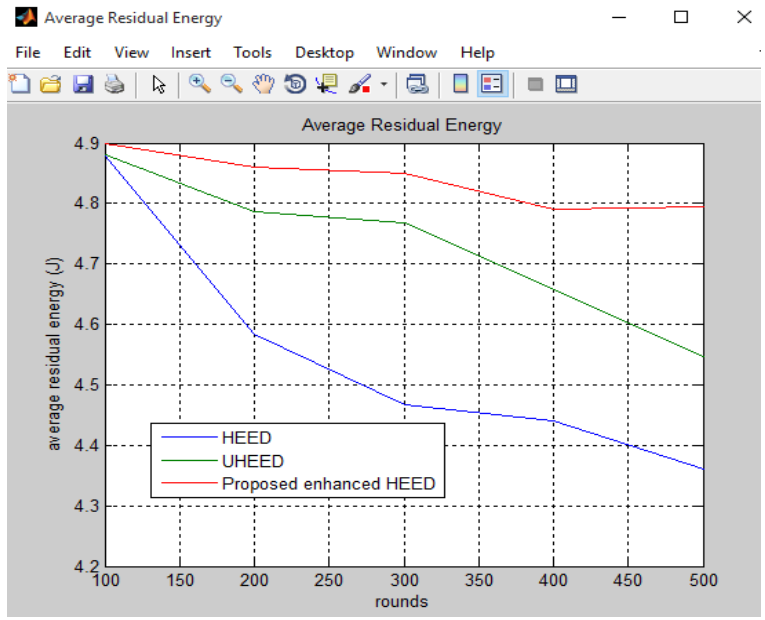

Fig 9: Average residual energy of the nodes versus rounds $(\mathrm{N}=50$ and $\mathrm{A}=100 \mathrm{~m} \times 100 \mathrm{~m})$

\section{CONCLUSIONS AND FUTURE SCOPE}

In this paper an enhancement of HEED is carried out by introducing the unequal clustering concept which resolves the hot spot issues not handled by HEED. The proposed enhanced HEED algorithm gives better performance compared to HEED and UHEED for the simulation scenario presented based on the different performance matrices like lifetime, average energy and the delay in packet delivery. Here the heterogeneity of the nodes is not considered. There are many unequal clustering techniques mentioned in the literature. As the future work, the comparative study on the influence of different unequal clustering in HEED will be embarked

\section{REFERENCES}

[1] Pantazis, N. a, Nikolidakis, S. a \& Vergados, D.D., 2013. Energy-efficient routing protocols in wireless sensor networks: A survey. Communications Surveys \& Tutorials, IEEE, 15(2), pp.551-591.

[2] Wang, J. et al., 2013. An energy efficient stable electionbased routing algorithm for wireless sensor networks. Sensors (Basel, Switzerland), 13(11), pp.14301-20. Available http://www.ncbi.nlm.nih.gov/pubmed/24887039.

[3] Vyas, P. \& Chouhan, M., 2014. Survey on Clustering Techniques in Wireless Sensor Network. , 5(5), pp.66146619.

[4] Selvi, G.V. \& Manoharan, R., 2013. A Survey of Energy Efficient Unequal Clustering Algorithms for Wireless Sensor Networks. , 79(1), pp.1-4.
[5] Wankhade, P.N.R. \& Choudhari, D.N., 2015. Energy Efficient Unequal Clustering Algorithm For Clustered Wireless., 3(3), pp.195-198.

[6] Younis, O. \& Fahmy, S., HEED : A Hybrid, EnergyEfficient, Distributed Clustering Approach for Ad-hoc Sensor Networks. , 0238294, pp.1-36.

[7] Lee, S. et al., 2008. An Energy-Efficient Distributed Unequal Clustering Protocol for Wireless Sensor Networks. , pp.443-447.

[8] Dhanpal, D.K., Joseph, A. \& Panicker, A., 2015. An Energy Efficient Unequal Cluster Based Routing Protocol For WSN With Non-Uniform Node Distribution. , 4(05).

[9] Chen, C. et al., 2015. IDUC: An Improved Distributed Unequal Clustering Protocol for Wireless Sensor Networks. , 2015.

[10] Hassan, S. \& Hamed, E., 2013. An Energy - Balancing Unequal Clustering Algorithm for Multi - hop Routing in WSN., (January).

[11] Kumar, A. \& Kumar, V., 2011. Energy Efficient Clustering and Cluster Head Rotation Scheme for Wireless Sensor Networks., 3(5), pp.129-136.

[12] Aierken, N. et al.,2015. RUHEED- Rotated Unequal Clustering Algorithm For Wireless Sensor Networks.

[13] Chen, J. \& Wang, N., 2010. Efficient Cluster Head Selection Methods for Wireless Sensor Networks. Sensors (Peterborough, NH), 5(8), pp.964-970.

[14] Mahajan, S., Malhotra, J. \& Sharma, S., 2014. An energy balanced QoS based cluster head selection strategy for WSN. Egyptian Informatics Journal, 15(3), pp.189-199. Available http://www.sciencedirect.com/science/article/pii/S11108 66514000322.

[15] Kumar, D., Aseri, T.C. \& Patel, R.B., 2010. Distributed Cluster Head Election ( DCHE ) Scheme for Improving Lifetime of Heterogeneous Sensor Networks. , 13(3), pp.337-348.

[16] Wang, L., Li, Y. \& Zhou, G., 2013. A Joint Weight Based Dynamic Clustering Algorithm for Wireless Sensor Networks *. , pp.325-335.

[17] A Alabass, K Elleithy, A.R., 2014. Dynamic Cluster Head Node Election (DCHNE) Model over Wireless Sensor Networks (WSNs). arXiv preprint arXiv:1410.5128. Available at http://khaledelleithy.org/Conferences/CATA 2014 paper 64.pdf.

[18] Murat Dener, 2014. Optimum Packet Length Over Data Transmission for Wireless Sensor Networks , pp.2-4. 